\title{
Applied Research of Computer Visual Arts on Digital Media in the Internet Age
}

\author{
Yan Hou
}

Qilu Normal University, Jinan, Shandong 250014, China.

\begin{abstract}
Since the reform and opening up, with the continuous development of the market economy and the advancement of science and technology, people have gradually strengthened the pursuit of the spiritual level while satisfying the material needs, and digital media have entered people's lives. The popularization and wide application of computer visual arts make science and technology integrated with each other, which not only bring the value of Internet technology into full play, but also enriches the artistic presentation of digital media, and satisfies people's spiritual needs in the era of diversified information. This article mainly focuses on the application of computer vision arts, analyzes its application principles and advantages, and looks into the future development trends of digital media arts by exploring specific application models, with a view to providing a theoretical basis for later related research.
\end{abstract}

Key words: Computer visual arts; Digital media; Application discussion.

\section{PRINCIPAL ANALYSIS OF COMPUTER VISUAL ARTS}

Digital media in this field is simply divided into two major categories: technology and art. Technology usually refers to the computer's hardware processing, and art to a certain extent is to meet people's pursuit of feeling, touch, and vision, which is a new situation that combines art and design.

Computer vision, also known as mechanical vision, simulates an optical system suitable for people's visual senses through various computer software, sensors and other devices based on computer technology, and then realizes the visualization of the object by light sources and optical systems and gives it intelligence.

Generally speaking, computer vision technology is generally widely used in the field of engineering technology. By using computer's image-assisted function, it can be set to meet the visual needs of the project and ensure that it can rely on the visual capabilities of the machine in later work, thus saving a great deal of manpower to a certain extent, improving the accuracy of the project, and embodying the value of computer vision art in digital media in the industrial field. Computer vision art involves a number of artistic fields and pays more attention to the artistry and aesthetics in the artistic process. Strengthening the use of computer visual arts in digital media, can not only improve the clarity of the picture, but also give people a new visual enjoyment and inspire people's pursuit of computer vision art. The practical basis of computer visual arts is to deal with images, and then pass the image through the analog pipeline to the signal in the form of a signal, in the transmission process, complete the processing measures, improve the picture quality and fluency at the same time, which enhances the art level and give people a new visual enjoyment, thereby stimulating people's pursuit of visual arts.

\section{ADVANTAGE ANALYSIS OF COMPUTER VISION ART IN DIGITAL MEDIA}

\section{Enhance the Infection of Artistic Vision}

Computer's visual arts involve many fields such as aesthetics and acoustics. To a certain extent, associating computers with life not only enriches the language form of computer visual arts, but also gives people a visual impact through dynamic expression patterns. It raises the overall level of art by infectious performances. Compared with the 
traditional two-dimensional art model, the computer visual arts have increased the color of art, reformed the traditional "black and white world", and enable people to mobilize the participation of multiple senses while enjoying beautiful images and inspire people's discovery of art. For example, virtual reality libraries and 5D cinemas are highly representative forms of art applications that have increased the infection of artistic videos.

\section{Enrich the Forms of Digital Media}

With the continuous development of computer electronic information technology, visual arts have also shown a good development trend. Under the current era of economic globalization and market dynamics, strengthening computer visual arts can not only improve the will interaction, but also enriches the form of digital media, which enriches people's production and life, and maximizes the degree of people's participation.

\section{Extend the Application Scope of Digital Media}

The integration of computer visual arts into the application of digital media expands the use of digital media to a certain extent, enriches the pursuit of spiritual levels, and improves the development of various types of application facilities. For example, the somatosensory function of smart phones, and the emergence and promotion of VR glasses illustrate the practical value of computer vision art and show that digital media has made a qualitative leap in the information age.

\section{Promote Enterprises' Economic Benefits}

While expanding the scope of digital media applications, computers have brought new opportunities for the development of enterprises. People have started to discuss the social value of digital media from different perspectives, so as to realize personal value and promote the economic benefits of enterprises. Specifically speaking, under the digital media of the new era, computer vision art can express abstract technologies in the form of figurative images to a certain degree, which will enrich people's lives, promote the artistic development of each person, and then promote the development of enterprises.

\section{ANALYSIS AND APPLICATION OF COMPUTER VISION ARTS IN DIGITAL MEDIA}

\section{Visual Art Application of Images}

Computer technology is used by computer visual art to deal with images, that is to say operate and process images. The image is simply a flat, two-dimensional art form. To integrate computer vision art into image processing, the first thing to understand is the structure layout, color ratio, and background of the image so that certain subjective intention can be added on the basis of understanding artistic conception and complete creation and processing of images. Through this "incorporated" art form, on the one hand, people's systematic understanding of images is enhanced, which can give them new artistic value according to their own emotions. On the other hand, it also promotes the development of image creation modes and improves traditional single form of artistic expression, thus fulfilling the diversified artistic needs of people in the current era, and accelerating the development of contemporary digital media art. At present, 3D video art performance form is the most common form of daily life is the. This art form is closer to people's real life, satisfies people's pursuit of images at the moment, and expresses images, words and sounds together, which enhance understanding and emotional advancement.

\section{Visual Art Application of Animation}

Most of the traditional animation is two-dimensional, which means it is expressed through the frame-by-frame art expression. The so-called frame-by-frame art expression is that the painter draws the scenes on the paper and then sorts it according to a certain time sequence. The related facilities of projection will be used to flip the paper to make it move so that it presents a continuous, logical new image to the audience. The traditional animation not only has the special beauty of Chinese culture and arts, but also integrates the vision for the future, making people appreciate and at the same time learn to establish a certain moral benchmark. For example, the early "Three Monks" was the most 
representative two-dimensional animation in China's early time. Through the form of animation, while satisfying people's spiritual pursuits, it also taught people the importance of "cooperation" and reflected social values. However, traditional animation usually requires high production costs and waste of resources, which is not conducive to the current principles of sustainable development in China. To ease the situation of resource shortage in the current era, scientific researchers integrate computer visual art into digital media. To a certain extent, the artistic expression of animation has been promoted, which has brought people a new visual impact, which improved the animation's expressiveness, enhanced the social value, and promoted the further development of digital media.

\section{Visual Art Application of Films and Television}

China's film and television industry was first started from Hong Kong, Macao and Taiwan, and then transited to inland China, which lasts 30-40 years. However, in the early stages of development, the film and television industry did not achieve much success. But with the continuous development of electronic information technology, the rise and widespread use of new media software in the 21 st century has also, to a certain extent, promoted the development of the film and television industry, greatly improved the market share of the enterprise and promoted the development of the market economy. In 2018, the cumulative total of the Spring Festival box office totaled more than one million, which increased 60 percent compared with last year. It can be said that with the help of the computer visual arts, it not only promoted the development of digital media technology, but also broke through the bottleneck of film and television industry in China and pushed the film and television industry to a higher position.

\section{Visual Art Application of Games}

The wide application of computer visual arts has not only promoted the development of traditional art forms such as images, animation, and film and television to a certain extent, but also has greatly broadened the application of digital media by applying computer technology visual arts to digital media, which has enabled it to inject new vitality into the game industry, brings new artistic and cultural feelings to people, enriches people's lives, and realizes the transition from traditional games to computer games, from computer-only games to high-speed visual games and improves the value of computer vision art in online games. From the initial 2D single-page online games to the vivid, fluency and exciting 3D three-dimensional games, it can be said that the games with computer visual arts are more in line with the diversified needs of contemporary people and enhances people's gaming experience. While passing a brand-new design concept to everyone, it brings people a new form of recreational entertainment that relieves social pressure. In order to ensure that computer visual arts are better integrated into digital media, relevant technology developers need to break the traditional innate thinking, establish a good game platform, and integrate themselves into the design of the game to create a more realistic and engaging game scenario to promote people's entertainment spirit. But it should be noted that the practice of these applications are based on healthy games, because with the rapid development of the network, internet likes a double-edged sword, bringing opportunities for digital media but also exacerbating the occurrence and spread of bad information. In the era of new media, people should strengthen the high-quality research and development of digital media, and then evade the emergence of shoddy games. At the same time, from the perspectives of vision, technology, humanization, and social responsibility, we should design games that can be played by all people and help young people realize the value of life.

\section{PROSPECT OF DIGITAL MEDIA ARTS}

With the continuous development of the market economy and advancement of electronic information technology, the information age has become an unavoidable development trend and digital media art has also penetrated into every aspect of life. As the so-called "science and technology are primary productive forces," in the course of the development of the next few years, the country will continue to focus on the research, development and exploration of computer technology, with a view to abandoning the drawbacks of traditional media technologies, guaranteeing the distinctive development of enterprises and ensuring the social value of the media.

Digital media not only shows in the digital design field, but also in many traditional art fields. It can be seen that the digital media with computer visual arts is no longer an unattainable performance art. It gradually develops in the direction of popularization, with a view to achieving the long-cherished wish of "inspired by life but beyond it". However, in the actual development process, the digital media art form also has certain drawbacks. It is manifested in the media workers' excessive attention to the development of the technical level but ignoring the social value and 
cultural connotation in the media, which has led to the development of the digital media only remaining in surface. It has not been able to penetrate art deeply, and it has only represented the presentation of technology in many fields, which has hindered the humanized development of digital media to some extent. Therefore, in order to ensure the sound development of digital media, in the process of integrating the computer visual arts, relevant management personnel must start from various aspects to ensure that the staff understand the connotation of the works, start from the cultural surface, and slowly infiltrate the internal culture value, giving it a deeper cultural heritage, so as to achieve a harmonious blend of various cultures, and then form a digital media art with a unique cultural heritage.

In China, the digital media based on the integration of art and technology has also developed over the past decades and has achieved certain achievements and some forms of art are well known in the world. In the future, the development of digital media needs to rely on the two forms of art and technology to ensure that they complement each other in the development process. At the same time, it should also integrate the traditional virtues of the Chinese nation with technological elements to enhance the digital media's h2umanization and emotionalization. At present, virtual technology is widely used in many areas such as $3 \mathrm{D}$, libraries, and cinemas in China. At the same time, its technology development level has reached the international advanced level standard. In the future development process, it will surely integrate with intelligent lifestyle to achieve the diversified needs of people under economic globalization, and to a certain extent, it can also change people's inherent ideological views on digital media and increase the overall awareness of it.

In short, the era of the future is the era of information technology, and the future of life is the life of intelligence. Under such an era, relevant scientific researchers must accelerate the speed of the research and development of related technologies in order to raise the level of humanization and emotionalization and ensure the function of the digital media fully realized.

\section{CONCLUSION}

Generally speaking, the development trend of the information age is unavoidable and it is not as simple as people think to integrate computer visual art into digital media. This kind of integration is not simply a sum of technology, but a brand-new creative method and technological concept. It plays an important role in advancing the development of digital media and enriching people's lives. Therefore, in the process of applying computer-vision art, relevant personnel can incorporate elements of "beauty" in the process of integration, thus ensuring the sound progress of digital media, playing a correct guiding role, and embodying social and cultural value to the greatest extent, thereby promoting the development of the enterprise.

\section{REFERENCES}

1. Liu Xiao, Wang Huixia. Application Research of Computer Visual Arts in the Digital Media Field [J]. China Internet, 2015(7): 121-124.

2. Hou Shoujin. New Media Art Design in Digital Era---Discussion of the Characters of New Media Art [J]. Art and Design (Theory), 2010(1): 123-124.

3. Sun Weite. Discussion on the Concept and Characteristics of Digital Media Industry [J]. Digital Fashion (New Vision Art), 2012(1):64-65.

4. Cong Cai. Discussion on the Application of Computer Vision Arts in Digital Media [J]. Electronic Production, 2013(18): 75-77. 